\title{
TRATAMENTO DE SEMENTES DE SORGO COM INSETICIDAS ${ }^{1}$
}

\author{
ALISSON VANIN² ${ }^{2}$ ALESSANDRO GUERRA DA SILVA ${ }^{3}$; CAMILA PEREIRA CAIXETA FERNANDES ${ }^{2}$, \\ WENDERSON SOUSA FERREIRA ${ }^{2}$, JUREMA FONSECA RATTES ${ }^{3}$
}

\begin{abstract}
RESUMO - O tratamento de sementes com inseticidas é um método eficiente que auxilia o manejo integrado de pragas, porém, há escassez de informações de produtos que apresentem seletividade para a cultura do sorgo. O objetivo deste trabalho foi o de avaliar a influência do tratamento de sementes de sorgo com inseticidas, contendo um ou dois princípios ativos, sem e com o armazenamento por 30 dias, na fase inicial de desenvolvimento das plantas. O experimento foi conduzido na Fesurv-Universidade de Rio Verde, em Rio Verde-GO com dez inseticidas (thiodicarb, fipronil, acephate, thiametoxam, imidacloprid, [imidacloprid+thiodicarb], imidacloprid+thiodicarb, imidacloprid+fipronil, thiametoxam+thiodicarb, thiametoxam+fipronil, acrescido da testemunha sem inseticida) sem e com o armazenamento das sementes por 30 dias. Os resultados obtidos permitiram constatar que o armazenamento por 30 dias das sementes de sorgo tratadas com inseticidas diminui o percentual de germinação. A mistura imidaclopridrid+thiodicarb apresenta maior potencial para uso no tratamento de sementes de sorgo. O inseticida acephate causa fitotoxicidade às plântulas de sorgo ao contrário do fipronil, thiametoxam e a mistura thiametoxam+thiodicarb que não prejudicam o vigor das sementes de sorgo.
\end{abstract}

Termos para indexação: Sorghum bicolor, fitotoxicidade, pragas.

\section{INSECTICIDE TREATMENT OF SORGHUM SEEDS}

\begin{abstract}
Seed treatment with insecticides is an effective tool in integrated pest management, but information on selective products to treat sorghum seeds is limited. The objective of this study was to evaluate the effects of treating sorghum seeds with insecticides containing one or two active ingredients, with and without storage for 30 days, on the early plant development. The experiment was conducted at Fesurv-Universidade de Rio Verde, in Rio Verde-GO, using ten insecticides (thiodicarb, fipronil, acephate, thiamethoxam, imidacloprid, [imidacloprid+thiodicarb], imidacloprid+thiodicarb, imidacloprid+fipronil, thiamethoxam+thiodicarb, thiamethoxam+fipronil, plus a non-insecticide control, with and without 30 day seed storage. The results showed that storage of sorghum seeds treated with insecticides for 30 days reduced the germination percentage. The imidacloprid+thiodicarb mixture has more potential for treating sorghum seeds. The insecticide acephate causes more phytotoxicity to the sorghum seedlings compared to fipronil, thiamethoxam and the thiamethoxam+thiodicarb mixture. The insecticides did not adversely affect sorghum seed vigor.
\end{abstract}

Index terms: Sorghum bicolor, phytotoxicity, pest.

${ }^{1}$ Submetido em 29/07/2010. Aceito para publicação em 11/10/2010.

${ }^{2}$ Eng. Agr., Mestrandos(as) em Produção Vegetal, Universidade de Rio Verde - FESURV, Rio Verde - GO, Caixa Postal 37, CEP: 75901970. e-mail: alissonvanin@hotmail.com; caixetacamila@hotmail.com; wendersonrv@hotmail.com.

${ }^{3}$ Eng. Agr., Professor(a) Doutor(a) da FESURV, Rio Verde, GO. e-mail: silvaag@yahoo.com.br; rattes@fesurv.br. 


\section{INTRODUÇÃO}

O sorgo é uma cultura amplamente cultivada na região Centro-Oeste em condições de safrinha (Coelho et al., 2002). Nesta região, o incremento na área de cultivo fez com que aumentasse a incidência de pragas, exigindo alternativas de controle para se evitar prejuízos no estabelecimento da cultura (Bleicher et al., 2003; Santos e Ávila, 2009). Neste contexto, a utilização de inseticidas no tratamento de sementes surge como uma alternativa promissora para minimizar os danos ocasionados pelas pragas de solo na fase inicial de desenvolvimento da cultura (Barros et al., 2001; Albajes et al., 2003) podendo ainda incrementar o rendimento das culturas ou reduzir os custos de produção (Smiderle e Cícero, 1998; Ceccon et al., 2004).

No milho, o tratamento de sementes com imidacloprid e thiametoxam apresentou eficiência no controle de adultos de cigarrinha (Dalbulus maydis, Hemiptera: Cicadellidae) até 30 após a emergência (Oliveira et al., 2008) e até 40 dias com uso do imidacloprid (Martins et al., 2008). Também os inseticidas clotianidina e thiametoxam, objetivando controle de Agrotis ipsilon (Hüfnagel) (Lepidoptera: Noctuidae) e Phyllophaga sp. (Coleoptera: Melolonthidae), não influenciaram o rendimento do milho (Wilde et al., 2007). O uso do imidacloprid em sementes de milho proporcionou menor incidência de Dichelops melacanthus (Hemiptera: Pentatomidae) (Martins et al., 2009). No trigo, o tratamento com imidacloprid proporcionou controle em adultos do pulgão verde e ninfas até 45 dias após a emergência (Costa et al., 2009). No sorgo, os inseticidas carbofuran e thiametoxam não foram eficientes aos 15 dias após a emergência para o controle de $S$. frugiperda, porém proporcionaram maior percentual de plântulas emergidas (Azevedo et al., 2004). Nessa cultura outros trabalhos têm comprovado a eficiência do tratamento de sementes com inseticidas no controle de pragas (Portillo et al., 1994; 1997).

O tempo de exposição das sementes aos inseticidas pode influenciar no estabelecimento das culturas. Tem-se constatado que o acephate, aldrin, carbofuran e thiodicarb podem ocasionar redução na germinação de sementes de milho quando a semeadura é realizada 10 dias após o tratamento, sendo que o inseticida carbofuran causou fitotoxicidade às sementes um dia após o tratamento (Oliveira e Cruz, 1986).

Os inseticidas clorpirifós e deltametrina não causaram efeitos na integridade de sementes de milho durante o período de armazenamento (Smiderle e Cícero, 1998). Entretanto, o carbofuran causou reduções significativas no vigor e na germinação de sementes de milho armazenadas por 15 ou 30 dias (Bittencourt et al., 2000). O armazenamento de sementes de feijão preto tratadas com thiametoxam e imidacloprid provocou redução na altura das plântulas provenientes destas (Guimarães et al., 2005). Segundo Horii et al. (2007) o inseticida thiametoxam pode promover efeitos no vigor das plântulas.

O uso de inseticidas para o tratamento de sementes de milho tem se mostrado como técnica promissora, existindo recomendação técnica para o uso desses (Portillo et al., 1994; 1997). No entanto, existem poucos estudos sobre os efeitos dos inseticidas utilizados no tratamento de sementes sobre o desenvolvimento inicial de plantas de sorgo. Diante disto, o objetivo deste trabalho foi avaliar a influência do tratamento de sementes de sorgo com inseticidas, contendo um ou dois princípios ativos, sem e com o armazenamento por 30 dias, na fase inicial de desenvolvimento das plantas.

\section{MATERIAL E MÉTODOS}

O ensaio foi conduzido em casa de vegetação, em canteiro de areia e no laboratório da Fesurv-Universidade de Rio Verde, em Rio Verde-GO de setembro a dezembro de 2008.

Em casa de vegetação, foram utilizados vasos de 8,01 , contendo $6,0 \mathrm{~kg}$ de subsolo classificado como Latossolo Vermelho distroférrico (Embrapa, 2006). As características químicas e físicas do solo foram: Ca: 0,09; Mg: 0,03; K: 0,02; Al: 0,01; H+Al: 0,7; Soma de bases: 0,13; CTC: 0,79, em $\mathrm{cmol}_{\mathrm{c}} \mathrm{dm}^{-3}$; P: $6 \mathrm{mg} \mathrm{dm}^{-3}$; matéria orgânica: $3,15 \mathrm{~g} \mathrm{~kg}^{-1} ; \mathrm{pH}\left(\mathrm{CaCl}_{2}\right)$ : 5,7; areia, silte e argila: 45; 1 ; e $54 \mathrm{~g} \mathrm{~kg}^{-1}$ em \%, respectivamente.

Para corrigir a baixa fertilidade do solo e suprir as necessidades da cultura do sorgo durante a condução do ensaio, foi efetuada a aplicação de calcário dolomítico dois meses antes da semeadura para elevar a saturação de bases a $60 \%$. Também foram empregados o equivalente à $10 \mathrm{~kg}$ ha $^{-1}$ de $\mathrm{N}$ na forma de sulfato de amônio na semeadura e 40 $\mathrm{kg} \mathrm{ha}^{-1}$ em cobertura aos quinze dias após a emergência. Também em semeadura foram empregados $260 \mathrm{~kg} \mathrm{ha}^{-1}$ de $\mathrm{P}_{2} \mathrm{O}_{5}$ na forma de superfosfato triplo e $140 \mathrm{~kg} \mathrm{ha}^{-1} \mathrm{de}$ $\mathrm{K}_{2} \mathrm{O}$ utilizando como fonte o cloreto de potássio. Este foi parcelado com a metade da dose aplicada na semeadura e o restante aos quinze dias após a emergência, junto com o N. 
O híbrido comercial de sorgo utilizado foi DKB 599, cujas sementes não tinham sido tratadas com inseticidas. Foi utilizado o delineamento experimental inteiramente casualizado, composto por quatro repetições, em esquema fatorial 2x11 referentes a sementes sem e com armazenamento por 30 dias, associadas a dez tratamentos de sementes, compostos por seis produtos comerciais, nas doses registradas para a cultura do milho.

Os tratamentos inseticidas utilizados foram: 1-thiodicarb (Futur 300): 0,600 kg de i.a. ha-1 $; 2$-fipronil (Standak): 0,025 kg de i.a. ha-1; 3-acephate (Acefato): 0,375 kg de i.a. ha-1; 4-thiametoxam (Cruiser $700 \mathrm{WS}$ ): 0,210 kg de i.a. ha ${ }^{-1} ; 5$-imidacloprid (Gaucho FS): $0,600 \mathrm{~kg}$ de i.a. ha-1 ${ }^{-1}$ 6-imidacloprid+thiodicarb (CropStar): 0,052 kg i.a. ha ${ }^{-1}+0,157$ kg de i.a. ha-1 ${ }^{-1}$ respectivamente; 4 prémisturas para aumentar o espectro de controle das pragas, com doses equivalentes a $60 \%$ da dose recomendada; 7-imidacloprid+thiodicarb: $0,360 \mathrm{~kg}$ i.a. $\mathrm{ha}^{-1}+0,360$ kg i.a. ha- ${ }^{-1}$, respectivamente; 8-imidacloprid+fipronil: $0,360 \mathrm{~kg}$ i.a. ha ${ }^{-1}+0,015 \mathrm{~kg}$ i.a. ha- ${ }^{-1}$, respectivamente; 9-thiametoxam+thiodicarb: $0,126 \mathrm{~kg}$ i.a. $\mathrm{ha}^{-1}+0,360 \mathrm{~kg}$ i.a. $\mathrm{ha}^{-1}$, respectivamente; 10-thiametoxam+fipronil: $0,126 \mathrm{~kg}$ i.a. ha-1 $+0,015 \mathrm{~kg}$ i.a. ha-1, respectivamente e um tratamento sem inseticida (testemunha), totalizando onze tratamentos inseticidas. No armazenamento por 30 dias após o tratamento com inseticidas, as sementes foram acomodadas em câmara fria a $16^{\circ} \mathrm{C}$ e UR de $50 \%$.

As características avaliadas foram: porcentagem de germinação e emergência de plântulas, altura de plantas, acúmulo de massa seca na parte aérea e raízes das plantas e fitotoxicidade aos 7,14 e 21 dias após a emergência (DAE) das plantas.

No laboratório foi avaliada a germinação das sementes de sorgo por meio da contagem de plântulas normais, conforme as Regras para Análise de Sementes (Brasil, 1992). Neste caso empregaram-se quatro repetições de 50 sementes, sendo as mesmas colocadas para germinar em substrato de papel de germinação (germitest) previamente umedecido em água. Utilizou-se quantidade de água destilada equivalente a 2,5 vezes a massa de papel seco, mantendo a temperatura de $25^{\circ} \mathrm{C}$.

A avaliação da emergência das plântulas de sorgo foi realizada em canteiro de leito de areia. Foram semeadas 200 sementes (quatro subamostras de 50 sementes), efetuando a contagem de plântulas emergidas aos 5 DAE, sendo os resultados convertidos em percentagem.

Em casa de vegetação e aos 45 DAE foram determinadas a altura de plantas (medição do colo até a inserção da última folha completamente desenvolvida), a massa seca da parte aérea e das raízes das plantas e os sintomas de fitotoxicidade após 7, 14 e 21 DAE. Foram semeadas oito sementes por vaso, sendo realizado o desbaste aos 7 DAE, deixando-se uma planta por vaso.

Para determinação da massa seca, foram coletadas separadamente a parte aérea e as raízes das plantas oriundas de cada tratamento, submetendo o material à secagem em estufa de circulação forçada de ar a $65{ }^{\circ} \mathrm{C}$ por $72 \mathrm{~h}$. Após atingir peso constante as amostras foram retiradas para determinação do peso seco da parte aérea e das raízes, expressando os resultados em gramas. Os sintomas de fitotoxicidade foram avaliados atribuindo-se notas visuais de 0 a 100 ( 0 para ausência de sintomas e 100 para morte das plantas) de acordo com recomendação da Alam (1974). No canteiro de areia e em casa de vegetação, as unidades experimentais foram irrigadas diariamente.

$\mathrm{Na}$ análise estatística, os dados referentes à percentagem de germinação, emergência das plântılas e de fitotoxicidade foram transformados para $\sqrt{x+1}$. Para todas as variáveis empregou-se análise de variância e quando constatada significância para determinada fonte de variação, empregou-se o teste de Tukey a 5\% de probabilidade para comparação das médias dos tratamentos. Efetuou-se também correlação entre as variáveis e toda a análise estatística foi realizada utilizando o software SISVAR (Ferreira, 2000).

\section{RESULTADOS E DISCUSSÃO}

$\mathrm{Na}$ análise de variância constatou-se significância para a interação inseticida $\mathrm{x}$ armazenamento para todas as variáveis analisadas, exceto para germinação e altura de plantas. Na avaliação da germinação, constatou-se significância para as fontes de variação inseticidas e armazenamento das sementes de sorgo (Tabela 1). A formulação [imidacloprid+thiodicarb] e a mistura de thiametoxam+thiodicarb ocasionaram menor percentual de germinação, diferindo dos tratamentos thiodicarb e imidacloprid+thiodicarb. Porém todos estes tratamentos inseticidas não diferiram da testemunha, demonstrando o potencial para uso no tratamento de sementes de sorgo. Resultados semelhantes foram obtidos em sementes de milho tratadas com thiodicarb e thiametoxam (Bittencourt et al., 2000; Ceccon et al., 2004).

Quanto ao efeito do período de armazenamento, constatou-se que as sementes que foram armazenadas por 30 dias apresentaram redução significativa na germinação 
(Tabela 1). Esta redução observada nas sementes que foram armazenadas pode ser atribuída à toxicidade dos inseticidas as plântulas, concordando com os resultados em sementes de milho obtidos por Oliveira e Cruz (1986). Por outro lado ausência de efeitos negativos na germinação foi constatada quando sementes de arroz foram tratadas com fipronil e armazenadas por 75 (Grutzmacher et al., 2008) e 90 dias (Veloso et al., 1988).

TABELA 1. Valores médios da germinação de sementes (GERM) e da altura de plantas (AP) de sorgo tratadas ou não com inseticidas com e sem armazenamento.

\begin{tabular}{lccc}
\hline Inseticidas & Dose (i.a. ha $\left.{ }^{-1}\right)$ & GERM $(\%)$ & AP (cm) \\
\hline thiodicarb & 0,600 & $91,00 \mathrm{a}$ & 52,75 \\
fipronil & 0,025 & $87,75 \mathrm{ab}$ & 52,63 \\
acephate & 0,375 & $89,00 \mathrm{ab}$ & 53,00 \\
thiametoxam & 0,210 & $89,25 \mathrm{ab}$ & 50,75 \\
imidacloprid & 0,600 & $84,25 \mathrm{ab}$ & 49,75 \\
[imidacloprid+thiodicarb] $^{1}$ & $0,052+0,157$ & $82,00 \mathrm{~b}$ & 49,75 \\
imidacloprid+thiodicarb & $0,360+0,360$ & $90,00 \mathrm{a}$ & 54,38 \\
imidacloprid+fipronil & $0,360+0,015$ & $86,25 \mathrm{ab}$ & 52,75 \\
thiametoxam+thiodicarb & $0,126+0,360$ & $82,25 \mathrm{~b}$ & 56,63 \\
thiametoxam+fipronil & $0,126+0,015$ & $88,50 \mathrm{ab}$ & 49,25 \\
\hline testemunha & 0 & $85,75 \mathrm{ab}$ & 51,63 \\
\hline C.V.(\%) & & 2,50 & 10,29 \\
\hline Armazenamento & & $89,62 \mathrm{a}$ & 53,16 \\
Sem armazenamento & & $84,18 \mathrm{~b}$ & 51,07 \\
Com armazenamento & & 2,72 & 10,01 \\
\hline C.V.(\%) & & & 5 \\
\hline
\end{tabular}

Médias seguidas pela mesma letra minúscula na coluna não diferem entre si pelo teste de Tukey em 5\%. ${ }^{1}$ Formulação comercial. ${ }^{2}$ Associação.

Para a altura de plantas, constatou-se ausência de significância para as fontes de variação testadas (Tabela 1), demonstrando que as plantas, independente do tratamento, apresentaram capacidade de recuperação até 45 DAE. No entanto, resultados de pesquisa comprovam a maior altura de plantas de milho com uso do fipronil no tratamento de sementes (Ceccon et al., 2004). A ausência de significância para altura de plantas de sorgo em função do período de armazenamento de sementes tratadas com inseticidas contrasta com os resultados obtidos por Guimarães et al. (2005), os quais constataram diferenças significativas em plantas de feijão preto, após tratamento com os inseticidas thiametoxam, thiodicarb e fipronil.
A emergência das plântulas de sorgo apresentou significância para a interação inseticida $\mathrm{x}$ período de armazenamento (Tabela 2). Quando as sementes não foram armazenadas, constatou-se que o maiorvalor de emergência foi obtido para a mistura do imidaclorpid+thiodicarb e o menor para o thiametoxam+thiodicarb. Porém, estes tratamentos não diferiram do valor obtido com a testemunha, indicando que os inseticidas não afetaram a emergência das plântulas. Ausência de significância foi observada quando as sementes foram armazenadas por 30 dias. Efeitos benéficos do armazenamento das sementes de sorgo na emergência das plântulas foram constatados com os inseticidas fipronil e com a mistura 
thiametoxam+fipronil. Os resultados obtidos contradizem aos obtidos por Dan et al. (2010) com uso do fipronil na germinação de sementes de soja. Em milho, redução no vigor das sementes tratadas com os inseticidas thiodicarb, thiametoxam e carbofuran armazenadas até 30 dias foram constatados por Bittencourt et al. (2000).

TABELA 2. Valores médios de emergência (EM), de acúmulo de massa seca da parte aérea (MSPA) e de raízes (MSRA) de plantas de sorgo tratadas ou não com inseticidas com e sem armazenamento.

\begin{tabular}{|c|c|c|c|}
\hline Inseticidas & Dose (i.a. ha ${ }^{-1}$ ) & Sem armazenamento & Com armazenamento \\
\hline \multicolumn{4}{|c|}{ - - - EM** $(\%)$ - - - } \\
\hline thiodicarb & 0,600 & $80,00 \mathrm{~A}$ ab & $89,00 \mathrm{~A} \mathrm{a}$ \\
\hline fipronil & 0,025 & $73,00 \mathrm{~B}$ ab & $90,00 \mathrm{~A} \mathrm{a}$ \\
\hline acephate & 0,375 & $84,50 \mathrm{~A} a b$ & $88,50 \mathrm{~A} \mathrm{a}$ \\
\hline thiametoxam & 0,210 & $81,00 \mathrm{~A}$ ab & $82,50 \mathrm{~A} \mathrm{a}$ \\
\hline imidacloprid & 0,600 & $78,00 \mathrm{~A}$ ab & $77,50 \mathrm{~A} \mathrm{a}$ \\
\hline [imidacloprid+thiodicarb] $^{1}$ & $0,052+0,157$ & $84,00 \mathrm{~A}$ ab & $72,50 \mathrm{~A}$ a \\
\hline imidacloprid+thiodicarb $^{2}$ & $0,360+0,360$ & $94,00 \mathrm{~A} \mathrm{a}$ & $77,50 \mathrm{~A} \mathrm{a}$ \\
\hline imidacloprid+fipronil & $0,360+0,015$ & $78,00 \mathrm{~A}$ ab & $76,50 \mathrm{~A} \mathrm{a}$ \\
\hline thiametoxam+thiodicarb & $0,126+0,360$ & $66,50 \mathrm{~A} \mathrm{~b}$ & $71,50 \mathrm{~A} \mathrm{a}$ \\
\hline thiametoxam+fipronil & $0,126+0,015$ & $68,50 \mathrm{~B} \mathrm{ab}$ & $95,50 \mathrm{~A} \mathrm{a}$ \\
\hline testemunha & 0 & $91,00 \mathrm{~A}$ ab & $92,50 \mathrm{~A} \mathrm{a}$ \\
\hline \multicolumn{4}{|c|}{ - - - MSPA** (g) - - } \\
\hline thiodicarb & 0,600 & $4,55 \mathrm{~A} \mathrm{ab}$ & $3,68 \mathrm{~B}$ ab \\
\hline fipronil & 0,025 & 3,88 A abcd & $3,25 \mathrm{~A} \mathrm{ab}$ \\
\hline acephate & 0,375 & 3,28 A bcde & $3,00 \mathrm{~A} \mathrm{ab}$ \\
\hline thiametoxam & 0,210 & $2,53 \mathrm{~B} \mathrm{e}$ & $3,43 \mathrm{~A} \mathrm{ab}$ \\
\hline imidacloprid & 0,600 & $2,55 \mathrm{Ade}$ & $2,53 \mathrm{~A} \mathrm{~b}$ \\
\hline [imidacloprid+thiodicarb] $^{1}$ & $0,052+0,157$ & $2,83 \mathrm{~A} \mathrm{de}$ & $3,00 \mathrm{~A} \mathrm{ab}$ \\
\hline imidacloprid+thiodicarb $^{2}$ & $0,360+0,360$ & $4,93 \mathrm{~A} \mathrm{a}$ & $4,15 \mathrm{~B} \mathrm{a}$ \\
\hline imidacloprid+fipronil & $0,360+0,015$ & $3,10 \mathrm{~B}$ cde & $3,88 \mathrm{~A} \mathrm{a}$ \\
\hline thiametoxam+thiodicarb & $0,126+0,360$ & $4,28 \mathrm{~A}$ abc & $3,95 \mathrm{~A} \mathrm{a}$ \\
\hline thiametoxam+fipronil & $0,126+0,015$ & 3,43 A bcde & $2,93 \mathrm{~A} a b$ \\
\hline testemunha & 0 & 2,98 A cde & $3,50 \mathrm{~A} \mathrm{ab}$ \\
\hline \multicolumn{4}{|c|}{ - - - MSRA** $(\mathrm{g})$ - - - } \\
\hline thiodicarb & 0,600 & $3,43 \mathrm{~A}$ a & $3,90 \mathrm{~A} \mathrm{~cd}$ \\
\hline fipronil & 0,025 & $3,95 \mathrm{~B} \mathrm{a}$ & $5,10 \mathrm{~A} \mathrm{BC}$ \\
\hline acephate & 0,375 & $3,35 \mathrm{~A}$ a & $3,03 \mathrm{~A} \mathrm{~d}$ \\
\hline thiametoxam & 0,210 & $3,23 \mathrm{~B}$ a & $5,58 \mathrm{~A} \mathrm{ab}$ \\
\hline imidacloprid & 0,600 & $3,33 \mathrm{~A} \mathrm{a}$ & $2,93 \mathrm{~A} \mathrm{~d}$ \\
\hline [imidacloprid+thiodicarb] $^{1}$ & $0,052+0,157$ & $2,95 \mathrm{~A} \mathrm{a}$ & $3,38 \mathrm{Ad}$ \\
\hline imidacloprid+thiodicarb $^{2}$ & $0,360+0,360$ & $3,30 \mathrm{~B} \mathrm{a}$ & $6,73 \mathrm{~A} \mathrm{a}$ \\
\hline imidacloprid+fipronil & $0,360+0,015$ & $3,55 \mathrm{~A} \mathrm{a}$ & $4,25 \mathrm{~A} \mathrm{bcd}$ \\
\hline thiametoxam+thiodicarb & $0,126+0,360$ & $4,15 \mathrm{~B}$ a & $5,15 \mathrm{~A}$ abc \\
\hline thiametoxam+fipronil & $0,126+0,015$ & $3,40 \mathrm{~A} \mathrm{a}$ & $3,10 \mathrm{~A} \mathrm{~d}$ \\
\hline testemunha & 0 & $3,40 \mathrm{~A} \mathrm{a}$ & $2,78 \mathrm{~A} \mathrm{~d}$ \\
\hline
\end{tabular}

Médias seguidas pela mesma letra minúscula na coluna e maiúscula na linha não diferem entre si pelo teste de Tukey em $5 \%$. ${ }^{1}$ Formulação comercial. ${ }^{2}$ Associação.**C.V. EM; MSPA; MSRA (\%): 7,02; 17,57; 16,91, respectivamente. 
Na avaliação do acúmulo da massa seca da parte aérea e raízes das plantas de sorgo, constataram-se significância para a interação inseticida $\mathrm{x}$ período de armazenamento (Tabela 2). Quando as sementes não foram submetidas ao armazenamento, o maior acúmulo de massa seca na parte aérea foi obtido com a mistura de imidaclorid+thiodicarb, seguido do thiodicarb e thiametoxam+thiodicarb. O menor valor foi observado para o thiametoxam, cujo resultado assemelhou-se ao da testemunha, comprovando desta forma a viabilidade do uso de inseticidas no tratamento de sementes. $\mathrm{O}$ mesmo pode ser comprovado no armazenamento por 30 dias, em que as misturas imidacloprid+thiodicarb, imidacloprid $+\mathrm{fi}$ pronil e thiametoxam+thiodicarb proporcionaram maior acúmulo de massa seca em relação ao imidacloprid.

Na comparação do acúmulo de massa seca da parte aérea para cada tratamento inseticida, constataramse menores valores quando as sementes foram armazenadas e tratadas com os inseticidas thiodicarb e imidacloprid+thiodicarb (Tabela 2). A partir dos resultados obtidos com esta mistura conclui-se que deve-se dar preferência ao uso da formulação comercial desses princípios ativos quando o objetivo é o maior acúmulo de massa seca na parte aérea das plantas de sorgo. Por outro lado, efeitos benéficos do armazenamento foram constatados com uso do thiametoxam e do imidacloprid+fipronil. Resultados semelhantes de massa seca da parte aérea aos 28 DAE em plantas de milho em relação à testemunha foram obtidos quando as sementes receberam os inseticidas thiamethoxam, carbofuran, imidacloprid+carbofuran, fipronil e thiodicarbe (Ceccon et al., 2004). Castro et al. (2008) também não encontraram diferenças significativas na massa seca de parte aérea aos $21 \mathrm{DAE}$ em soja com uso dos inseticidas thiametoxam e imidacloprid em relação à testemunha.

$\mathrm{Na}$ análise da massa seca da raiz, constatou-se ausência de significância entre os tratamentos inseticidas sem o armazenamento das sementes (Tabela 2). Com o armazenamento, foi observado efeito benéfico no acúmulo de massa seca das raízes com a mistura imidacloprid+thiodicarb, seguido do thiametoxam, thiametoxam+thiodicarb e fipronil. Para os demais tratamentos não foi constatada diferença significativa para o acúmulo de massa seca de raízes. Isto demonstra o potencial de utilização dos inseticidas para a cultura do sorgo, principalmente para produção de raízes, suportando condições de deficiência hídrica durante o cultivo de safrinha (Coelho et al., 2002; Marin et al., 2006; Silva et al., 2009). A maior produção de raízes proporcionada por esses inseticidas são semelhantes aos obtidos por Lauxen et al.
(2010) em algodão quando as sementes foram tratadas com thiametoxam. Porém, efeitos negativos no comprimento das raízes e de plântulas foram observados em sementes de soja tratadas com fipronil, acefato e thiametoxam (Dan et al., 2010). No trabalho, valores semelhantes ao da testemunha foram obtidos com o acephate, imidacloprid, [imidacloprid+thiodicarb] e thiametoxam+fipronil. De qualquer forma, como também verificado na avaliação da massa seca da parte aérea, os resultados obtidos com a massa seca de raízes de sorgo comprovam a viabilidade do uso de inseticidas no tratamento de sementes. Os resultados obtidos estão em concordância com os de Castro et al. (2008) que não encontraram diferença significativa para os tratamentos thiametoxam e imidacloprid em relação à testemunha, em raízes de soja.

Pode-se inferir que o vigor das sementes não foi afetado quando as sementes não foram submetidas ao armazenamento, constatando ausência de diferenças significativas entre os valores obtidos de emergência e massa seca de raízes das sementes tratadas com a testemunha. As sementes tratadas com os inseticidas thiodicarb e imidacloprid+thiodicarb apresentaram valores superiores de massa seca de parte aérea em relação a testemunha. Quando realizado o armazenamento, observouse que a mistura imidacloprid+thiodicarb, thiametoxam, thiametoxam+thiodicarb e fipronil proporcionou maior massa seca de raízes em relação a testemunha. Nessa condição, os valores obtidos de emergência e massa seca da parte aérea das sementes tratadas com inseticidas apresentaram resultados semelhantes ao da testemunha. Sendo assim, pode-se inferir que o tratamento de sementes com inseticidas não prejudicou o vigor das sementes, visto que os resultados obtidos com o tratamento de sementes de sorgo foram semelhantes ou superiores aos observados com o da testemunha.

$\mathrm{Na}$ avaliação da fitotoxicidade (7, 14 e 21 DAE) verificou-se que os tratamentos inseticidas, associados ao tempo de armazenamento, ocasionaram clorose nas plantas de sorgo. Em todas as épocas de avaliação, constatou-se significância para essas fontes de variação (Tabela 3). O uso do inseticida acephate ocasionou os maiores valores de fitotoxicidade, independente do armazenamento das sementes. Quando se realizou a semeadura do sorgo logo após o tratamento das sementes constatou-se que os menores valores de fitotoxicidade aos 21 DAE foram observados com uso do fipronil e thiametoxam, aliado à mistura thiametoxam+thiodicarb, cujos resultados assemelharam-se ao da testemunha. 
Resultados semelhantes foram obtidos por Bittencourt da cultura com sementes tratadas com inseticidas deve et al. (2000) em milho, constatando que a implantação ser, de preferência, o mais breve possível.

TABELA 3. Valores médios do teste padrão fitotoxicidade aos 7, 14 e 21 dias de emergência das plantas de sorgo tratadas ou não com inseticidas com e sem armazenamento.

\begin{tabular}{|c|c|c|c|}
\hline Inseticidas & Dose (i.a. ha ${ }^{-1}$ ) & Sem armazenamento & Com armazenamento \\
\hline \multicolumn{4}{|c|}{ - - - 7 DAE $(\%)$ - - - } \\
\hline thiodicarb & 0,600 & $9,50 \mathrm{~B}$ abc & $13,75 \mathrm{~A} \mathrm{bc}$ \\
\hline fipronil & 0,025 & $5,00 \mathrm{~B} \mathrm{~d}$ & 8,75 A cde \\
\hline acephate & 0,375 & $12,50 \mathrm{~B}$ a & $21,25 \mathrm{~A} \mathrm{a}$ \\
\hline thiametoxam & 0,210 & $6,50 \mathrm{~A} \mathrm{bcd}$ & $8,25 \mathrm{Ade}$ \\
\hline imidacloprid & 0,600 & $9,00 \mathrm{~B}$ abcd & $14,25 \mathrm{~A} \mathrm{~b}$ \\
\hline${\text { [imidacloprid+thiodicarb }]^{1}}^{1}$ & $0,052+0,157$ & $10,50 \mathrm{~B}$ ab & $15,00 \mathrm{~A} \mathrm{ab}$ \\
\hline imidacloprid+thiodicarb $^{2}$ & $0,360+0,360$ & 7,50 B bcd & $13,00 \mathrm{~A} \mathrm{bcd}$ \\
\hline imidacloprid+fipronil & $0,360+0,015$ & $5,75 \mathrm{~A} \mathrm{~cd}$ & $7,50 \mathrm{~A} \mathrm{e}$ \\
\hline thiametoxam+thiodicarb & $0,126+0,360$ & $6,25 \mathrm{~B} \mathrm{bcd}$ & $8,50 \mathrm{Ade}$ \\
\hline thiametoxam+fipronil & $0,126+0,015$ & $6,50 \mathrm{~A} \mathrm{bcd}$ & $5,75 \mathrm{~A} \mathrm{e}$ \\
\hline testemunha & 0 & $0,00 \mathrm{~A} \mathrm{e}$ & $0,00 \mathrm{~A} \mathrm{f}$ \\
\hline \multicolumn{4}{|c|}{ - - 14 DAE $(\%)-\ldots$} \\
\hline thiodicarb & 0,600 & $8,50 \mathrm{~B} \mathrm{abc}$ & $11,75 \mathrm{~A} \mathrm{bc}$ \\
\hline fipronil & 0,025 & $4,00 \mathrm{~B} \mathrm{~d}$ & $7,25 \mathrm{~A}$ de \\
\hline acephate & 0,375 & $10,75 \mathrm{~B}$ a & $17,75 \mathrm{~A} \mathrm{a}$ \\
\hline thiametoxam & 0,210 & $5,00 \mathrm{~A} \mathrm{~cd}$ & $7,00 \mathrm{~A} \mathrm{e}$ \\
\hline imidacloprid & 0,600 & $10,00 \mathrm{~B} \mathrm{ab}$ & $15,00 \mathrm{~A} \mathrm{ab}$ \\
\hline [imidacloprid+thiodicarb] $^{1}$ & $0,052+0,157$ & $8,50 \mathrm{~A}$ abc & $11,25 \mathrm{~A} \mathrm{bcd}$ \\
\hline imidacloprid+thiodicarb $^{2}$ & $0,360+0,360$ & 7,25 A abcd & $7,25 \mathrm{Ade}$ \\
\hline imidacloprid+fipronil & $0,360+0,015$ & $5,75 \mathrm{~A} \mathrm{~cd}$ & 8,25 A cde \\
\hline thiametoxam+thiodicarb & $0,126+0,360$ & $5,00 \mathrm{~A} \mathrm{~cd}$ & $5,25 \mathrm{~A} \mathrm{e}$ \\
\hline thiametoxam+fipronil & $0,126+0,015$ & $6,50 \mathrm{~A} \mathrm{bcd}$ & $5,00 \mathrm{~A} \mathrm{e}$ \\
\hline testemunha & 0 & $0,00 \mathrm{~A} \mathrm{e}$ & $0,00 \mathrm{~A} \mathrm{f}$ \\
\hline \multicolumn{4}{|c|}{ - - - 21 DAE (\%) - - } \\
\hline thiodicarb & 0,600 & $3,50 \mathrm{~B} \mathrm{~b}$ & $7,25 \mathrm{~A} \mathrm{bcd}$ \\
\hline fipronil & 0,025 & $0,00 \mathrm{~B} \mathrm{c}$ & $3,50 \mathrm{~A} \mathrm{e}$ \\
\hline acephate & 0,375 & $8,50 \mathrm{~B} \mathrm{a}$ & $15,75 \mathrm{~A} \mathrm{a}$ \\
\hline thiametoxam & 0,210 & $0,75 \mathrm{~B} \mathrm{c}$ & $4,25 \mathrm{~A}$ de \\
\hline imidacloprid & 0,600 & $6,50 \mathrm{~B}$ ab & $11,00 \mathrm{~A} \mathrm{ab}$ \\
\hline${\text { [imidacloprid+thiodicarb }]^{1}}^{1}$ & $0,052+0,157$ & $4,00 \mathrm{~B} \mathrm{~b}$ & $8,50 \mathrm{~A} \mathrm{bc}$ \\
\hline imidacloprid+thiodicarb $^{2}$ & $0,360+0,360$ & $5,25 \mathrm{~A} \mathrm{ab}$ & $3,75 \mathrm{~A}$ de \\
\hline imidacloprid+fipronil & $0,360+0,015$ & $4,50 \mathrm{~A} a b$ & $5,25 \mathrm{~A}$ cde \\
\hline thiametoxam+thiodicarb & $0,126+0,360$ & $0,00 \mathrm{~B} \mathrm{c}$ & $3,50 \mathrm{Ade}$ \\
\hline thiametoxam+fipronil & $0,126+0,015$ & $4,00 \mathrm{~A} \mathrm{~b}$ & $4,00 \mathrm{Ade}$ \\
\hline testemunha & 0 & $0,00 \mathrm{~A} \mathrm{c}$ & $0,00 \mathrm{~A} \mathrm{f}$ \\
\hline
\end{tabular}

Médias seguidas pela mesma letra minúscula na coluna e maiúscula na linha não diferem entre si pelo teste de Tukey em $5 \%$. ${ }^{1}$ Formulação comercial. ${ }^{2}$ Associação. ${ }^{* *}$ C.V. 7DAE; 14DAE; 21DAE (\%): 11,39; 10,31; 16,93, respectivamente. 
O armazenamento das sementes por 30 dias proporcionou, aos $21 \mathrm{DAE}$, em relação ao período sem o armazenamento, maior fitotoxicidade com uso dos inseticidas thiodicarb, fipronil, acephate, thiametoxam, imidacloprid, [imidacloprid+thiodicarb] e da mistura thiametoxam+thiodicarb (Tabela 3). Destes apenas o acephate e o imidacloprid ocasionaram fitotoxicidade maior que $10 \%$, porém, com valores menores em relação à primeira época de avaliação. Verificou-se que em todos os tratamentos os sintomas diminuíram com o tempo, demonstrando a recuperação das plantas de sorgo ao efeito fitotóxico dos inseticidas.

A viabilidade do tratamento de sementes de sorgo com inseticidas pode ser comprovada também pela análise de correlação das características avaliadas (Tabela 4). Sem o armazenamento das sementes, nota-se que a altura de plantas de sorgo correlacionou positiva e significativamente com o acúmulo de massa seca na parte aérea e raízes das plantas. Destaca-se também a correlação negativa e significativa entre a massa seca de raiz e a fitotoxicidade aos 21 DAE. O mesmo ocorreu para a variável massa seca da parte aérea, quando as sementes foram armazenadas por 30 dias. Portanto constata-se que a fitotoxicidade provocada pelos inseticidas influenciou negativamente no acúmulo de massa seca de raízes, além da parte aérea quando as sementes foram armazenadas. Destaca-se também que a germinação não apresentou correlação significativa com nenhuma das variáveis. Os resultados novamente comprovam a viabilidade do tratamento de sementes de sorgo com inseticidas, concordando com outros trabalhos de pesquisa na cultura do milho (Portillo et al., 1997; Bittencourt et al., 2000; Ceccon et al., 2004).

TABELA 4. Coeficientes de Correlação de Pearson para as variáveis, emergência de plântulas (EM), germinação de sementes (GERM), altura de plantas (AP), fitotoxicidade aos 7, 14 e 21 dias após emergência (DAE) e acúmulo de massa seca nas raízes (MSRA) e parte aérea (MSPA) de plantas de sorgo provenientes de sementes tratadas ou não com inseticidas, sem e com armazenamento.

\begin{tabular}{|c|c|c|c|c|c|c|c|c|}
\hline VAR. & EM & GERM & AP & 7DAE & 14DAE & 21DAE & MSRA & MSPA \\
\hline \multicolumn{9}{|c|}{--- Sem armazenamento --- } \\
\hline EM & - & & & & & & & \\
\hline GERM & $-0,14^{\mathrm{ns}}$ & - & & & & & & \\
\hline $\mathrm{AP}$ & $-0,18^{\mathrm{ns}}$ & $-0,001^{\mathrm{ns}}$ & - & & & & & \\
\hline 7DAE & $0,04^{\mathrm{ns}}$ & $-0,07^{\mathrm{ns}}$ & $-0,11^{\mathrm{ns}}$ & - & & & & \\
\hline 14DAE & $0,08^{\mathrm{ns}}$ & $0,2^{\mathrm{ns}}$ & $-0,006^{\mathrm{ns}}$ & $0,83 * *$ & - & & & \\
\hline 21DAE & $0,15^{\mathrm{ns}}$ & $0,06^{\mathrm{ns}}$ & $-0,06^{\mathrm{ns}}$ & $0,62 * *$ & $0,74 * *$ & - & & \\
\hline MSRA & $-0,23^{\mathrm{ns}}$ & $-0,12^{\text {ns }}$ & $0,37 *$ & $-0,17^{\mathrm{ns}}$ & $-0,21^{\mathrm{ns}}$ & $-0,29 *$ & - & \\
\hline MSPA & $-0,04^{\mathrm{ns}}$ & $0,004^{\mathrm{ns}}$ & $0,53 * *$ & $-0,03^{\text {ns }}$ & $0,03^{\mathrm{ns}}$ & $-0,01^{\mathrm{ns}}$ & $0,30 *$ & - \\
\hline \multicolumn{9}{|c|}{--- Com armazenamento --- } \\
\hline EM & - & & & & & & & \\
\hline GERM & $0,15^{\mathrm{ns}}$ & - & & & & & & \\
\hline AP & $-0,11^{\mathrm{ns}}$ & $-0,03^{\mathrm{ns}}$ & - & & & & & \\
\hline 7DAE & $-0,25^{\text {ns }}$ & $0,07^{\mathrm{ns}}$ & $0,01^{\mathrm{ns}}$ & - & & & & \\
\hline 14DAE & $-0,17^{\mathrm{ns}}$ & $-0,02^{\mathrm{ns}}$ & $0,02^{\mathrm{ns}}$ & $0,90 * *$ & - & & & \\
\hline $21 \mathrm{DAE}$ & $-0,12^{\mathrm{ns}}$ & $-0,07^{\mathrm{ns}}$ & $-0,01^{\mathrm{ns}}$ & $0,82 * *$ & $0,89 * *$ & - & & \\
\hline MSRA & $-0,14^{\mathrm{ns}}$ & $0,14^{\mathrm{ns}}$ & $0,22^{\mathrm{ns}}$ & $-0,03^{\mathrm{ns}}$ & $-0,18^{\mathrm{ns}}$ & $-0,31^{*}$ & - & \\
\hline MSPA & $-0,07^{\mathrm{ns}}$ & $-0,05^{\mathrm{ns}}$ & $0,16^{\mathrm{ns}}$ & $-0,22^{\mathrm{ns}}$ & $-0,29^{\mathrm{ns}}$ & $-0,35^{*}$ & $0,60 * *$ & - \\
\hline
\end{tabular}

ns, **, * - Não significativo, significativo em 1,0 e $5,0 \%$ de probabilidade pelo teste $\mathrm{F}$, respectivamente.

De acordo com os resultados obtidos, podese inferir que os tratamentos fipronil, thiametoxam, thiametoxam+thiodicarb e imidacloprid+thiodicarb são adequadosparaotratamentodesementes desorgocomousem 
oarmazenamentopor30dias. Diantedisto, ressalta-sequeasmisturas de inseticidas (imidacloprid+thiodicarb, imidacloprid+fipronil, thiametoxam+thiodicarb e thiametoxam+fipronil) apresentaram menor fitotoxicidade as plantas de sorgo.

\section{CONCLUSÕES}

O armazenamento por 30 dias das sementes de sorgo tratadas com inseticidas influencia negativamente a germinação.

A mistura imidacloprid+thiodicarb apresenta maior potencial para uso no tratamento de sementes de sorgo.

$\mathrm{O}$ inseticida acephate causa maior fitotoxicidade às plântulas de sorgo ao contrário do fipronil, thiametoxam e a mistura thiametoxam+thiodicarb.

Os inseticidas não prejudicam o vigor das sementes de sorgo.

\section{REFERÊNCIAS}

ASOCIOCIÓN LATINOAMERICANA DE MALEZAS. Recomendaciones sobre unificación de los sistemas de evaluación en ensayos de control de malezas. ALAM, v.1, n.1, p.35-38, 1974.

ALBAJES, R.; LÓPEZ, C.; PONS, X. Predatory fauna in cornfields and response to imidacloprid seed treatment. Journal of Economic Entomology, v.96, n.6, p.18051813,2003.http://www.bioone.org/doi/abs/10.1603/00220493-96.6.1805?journalCode=ecen

AZEVEDO, R. de; GRUTZMACHER, A.D.; LOECK, A.E.; SILVA, F.F. DA; STORCH, G.; HERPICH, M.I. Efeito do tratamento de sementes e aplicações foliares de inseticidas em diferentes volumes de calda, no controle de Spodoptera frugiperda (J.E. Smith, 1797) (Lepidoptera: Noctuidae), nas culturas do milho e sorgo em agroecossistema de várzea. Revista Brasileira de Agrociência, v.10, n.1, p.71-77, 2004. http://www.ufpel. tche.br/faem/agrociencia/v10n1/artigo10.pdf

BARROS, R.G.; YOKOYAMA, M.; COSTA, J.L. da S. Compatibilidade do inseticida Thiamethoxam com fungicidas utilizados no tratamento de sementes de feijoeiro. Pesquisa Agropecuária Tropical, v.31, n.2, p.153-157, 2001. http://scholar.google.com.br/ scholar?hl $=$ pt-BR\& $\&=\% 22$ Compatibilidade + do + inseti $\mathrm{cida}+$ Thiamethoxam $+\mathrm{com}+$ fungicidas + utilizados $+\mathrm{no}+\mathrm{t}$ ratamento $+\mathrm{de}+$ sementes + de + feijoeiro. $+\% 22 \& 1 r=\&$ as ylo $=\&$ as $\_$vis $=0$

BITTENCOURT, S.R.M. de; FERNANDES, M.A.;
RIBEIRO, M.C.; VIEIRA, R.D. Desempenho de sementes de milho tratadas com inseticidas sistêmicos. Revista Brasileira de Sementes, v.22, n.2, p.86-93, 2000. http:// www.abrates.org.br/revista/artigos/2000/v22n2/artigo12. pdf

BLEICHER, E.; OLIVEIRA, I.S.R. de; VIDAL NETO, F. das C. Controle da lagarta-do-cartucho do milho usando-se areia como veículo de inseticida. Revista Ciência Agronômica, v.34, n.1, p.5-10, 2003.http://www.ccarevista.ufc.br/site/artigos_lista. php?sel $=2003 \&$ sel $2=1 \&$ sel $3=34$

BRASIL. Ministério da Agricultura e Reforma Agrária. Secretaria NacionaldeDefesa Agropecuária.Departamento Nacional de Produção Vegetal. Coordenação de Laboratório Vegetal. Regras para Análise de Sementes. Brasília, DF, 1992. 365p.

CASTRO, G.S.A.; BOGIANI, J.C.; SILVA, M.G. da; GAZOLA, E.; ROSOLEM, C.A. Tratamento de sementes de soja com inseticidas e um bioestimulante. Pesquisa Agropecuária Brasileira, v.43, n.10, p.13111318, 2008.http://www.scielo.br/scielo.php?pid=S0100204X2008001000008\&script=sci_arttext\&tlng=e

CECCON, G.; RAGA, A.; DUARTE, A.P.; SILOTO, R.C. Efeito de inseticidas na semeadura sobre pragas iniciais e produtividade de milho safrinha em plantio direto. Bragantia, v.63, n.2, p.227-237, 2004. http:// www.scielo.br/pdf/brag/v63n2/21372.pdf

COELHO, A.M.; WAQUIL, J.M.; KARAN, D.; CASELA, C.R.; RIBAS, P.M. Seja o doutor do seu sorgo. Informações Agronômicas, Piracicaba, n.100, 2002. 24p. (Arquivo do Agrônomo, 14). http://www.cnpms. embrapa.br/sorgo/doutorsorgo.pdf

COSTA, R.R.; MORAES, J.C.; COSTA, R.R. da. Interação silício-imidacloprid no comportamento biológico e alimentar de Schizaphis graminum (Rond.) (Hemiptera: Aphididae) em plantas de trigo. Ciência e Agrotecnologia, v.33, n.2, p.455-460, 2009. http://www.scielo.br/scielo. php?pid=S1413-70542009000200014\&script $=$ sci abstract\&tlng=pt

DAN, L.G. de M.; DAN, H. de A.; BARROSO, A.L. de L.; BRACCINI, A. de L. Qualidade fisiológica de sementes de soja tratadas com inseticidas sob efeito do armazenamento. Revista Brasileira de Sementes, v.32, n.2, p.131-139, 2010.

EMBRAPA. Centro Nacional de Pesquisa de solos. Sistema de classificação de solos. 2.ed. Rio de Janeiro: Embrapa Solos, 2006. 306p. 
FERREIRA, D.F. Analises estatísticas por meio do Sisvar para Windows 4.0. In: REUNIAO ANUAL DA REGIAO BRASILEIRA DA SOCIEDADE INTERNACIONAL DE BIOMETRIA, 45, 2000, São Carlos, Anais... São Carlos: UFSCar, 2000. p.255-258.

GRUTZMACHER, A.D.; MARTINS, J.F. da S.; CUNHA, U.S. da; GIOLO, F.P.; NEVES, M.B. das; HARTER, W. da R.; FRANCO, D.F.; MATTOS, M.L.T. Viabilidade da antecipação do tratamento de sementes de arroz com inseticidas em relação à data de semeadura no controle de Oryzophagus oryzae (Coleoptera: Curculionidae). Ciência Rural, v.38, n.7, p.1830-1835, 2008. http:// www.scielo.br/pdf/cr/v38n7/a05v38n7.pdf

GUIMARÃES, R.N.; PORTO, T.B.; PEREIRA, J.M.; BARBOSA, L. de A.; FERNANDES, P.M.; COSTA, R.B. da; BARROS, R.G. Efeito do tratamento de sementes com inseticidas na emergência e altura de plântulas de feijão. In: CONGRESSO NACIONAL DE PESQUISA DE FEIJÃO, 8., 2005, Goiânia. Anais... Santo Antônio de Goiás: Embrapa Arroz e Feijão, 2005. p.94-99. http:// www.cnpaf.embrapa.br/conafe/pdf/conafe2005-0342.pdf

HORII, A.; McCUE, P.; SHETTY, K. Enhancement of seed vigour following insecticide and phenolic elicitor treatment. Bioresource Technology. v.98, n.3, p.623632, 2007.

LAUXEN, L.R.; VILLELA, F.A.; SOARES, R.C. Desempenho fisiológico de sementes de algodoeiro tratadas com tiametoxam. Revista Brasileira de Sementes, v.32, n.3, p.61-68, 2010. http://www.scielo.br/ $\mathrm{pdf} / \mathrm{rbs} / \mathrm{v} 32 \mathrm{n} 3 / \mathrm{v} 32 \mathrm{n} 3 \mathrm{a} 07 . \mathrm{pdf}$

MARIN, F.R. et al. Perda de produtividade potencial da cultura do sorgo no Estado de São Paulo. Bragantia, v.65, n.1, p.157-162, 2006. http://www.scielo.br/pdf/ brag/v65n1/29050.pdf

MARTINS, G.M.; TOSCANO, L.C.; TOMQUELSKI, G.V.; MARUYAMA, W.I. Eficiência de inseticidas no controle de Dalbulus maidis (Hemiptera: Cicadellidae) na cultura do milho. Revista Caatinga, v.21, n.4, p.196-200, 2008. http://periodicos.ufersa.edu.br/index.php/sistema/ article/view/425/430

MARTINS, G.L.M.; TOSCANO, L.C.; TOMQUELSKI, G.V.; MARUYAMA, W.I. Controle químico do percevejo barriga-verde Dichelops melacanthus (Hemiptera: Pentatomidae) na cultura do milho. Arquivo do Instituto Biológico, v.76, n.3, p.475-478, 2009. http://www. biologico.sp.gov.br/docs/arq/v76_3/martins.pdf

OLIVEIRA, L.J.; CRUZ, I. Efeito de diferentes inseticidas e dosagens na germinação de sementes de milho. Pesquisa Agropecuária Brasileira, v.21, n.6, p.579-585, 1986. http://orton.catie.ac.cr/cgi-bin/wxis. exe/?IsisScript $=$ ACERVO. $x$ is $\&$ method $=$ post $\&$ formato $=2$ \& cantidad $=1$ \& expresion $=\mathrm{mfn}=023768$

OLIVEIRA, C.M. de; OLIVEIRA, E. de; CANUTO, M.; CRUZ, I. Eficiência de inseticidas em tratamento de sementes demilhonocontroledacigarrinhaDalbulusmaidis (Hemiptera: Cicadellidae) em viveiro telado. Ciência Rural, v.38, n.1, p.231-235, 2008. http://www.scielo.br/ scielo.php?pid=S0103-84782008000100037\&script $=$ sci_ abstract\&tlng $=\mathrm{pt}$

PORTILLO, H.E.; PITRE, H.N.; MECKENSTOCK, D.H. et al. Improved chemical protection of sorghum seed and seedlings from insect pests in Honduras. Turrialba, v.44, n.1, p.50-55, 1994. http://orton.catie.ac.cr/repdoc/ A0793e/A0793e01.html

PORTILLO, H.E.; PITRE, H.N.; MECKENSTOCK, D.H.; GÓMEZ, F.; LÓPEZ, J.I. Validation of new agronomic plant protection technologies in intercropped sorghum and maize in southern Honduras. Ceiba, v.38, n.1, p.35-43, 1997. http://orton.catie.ac.cr/cgi-bin/wxis. exe/?IsisScript $=$ GREYLIT .xis $\&$ method $=$ post $\&$ formato $=$ $2 \&$ cantidad $=1$ \&expresion $=\mathrm{mfn}=017712$

SANTOS, V.; AVILA, C.J. Aspectos Biológicos e Comportamentais de Liogenys suturalis Blanchard (Coleoptera: Melolonthidae) no Mato Grosso do Sul. Neotropical Entomology, v.38, n.6, p.734-740, 2009. http://www.scielo.br/scielo.php?pid=S1519-566X20090 $00600005 \&$ script $=$ sciarttext\&tlng $=$ en

SILVA, A.G. da; BARROS, A.S.; SILVA, L.H.C.P. da; SILVA, G.P. da; MORAES, E.B. de; PIRES, R.; TEIXEIRA, I.R. Avaliação de cultivares de sorgo granífero na safrinha no sudoeste do Estado de Goiás. Pesquisa Agropecuária Tropical, v.39, n.2, p.168174, 2009. http://revistas.ufg.br/index.php/pat/article/ viewArticle/3320

SMIDERLE, O.J.; CICERO, S.M. Tratamento inseticida e qualidade de sementes de milho. Revista Brasileira de Sementes, v.20, n.2, p.223-230, 1998. http://www. abrates.org.br/revista/artigos/1998/v20n2/artigo36.pdf

VELOSO, V. da R.S.; SILVA, A.L. da; GUIMARÃES, N.N.R. Efeito de diferentes inseticidas na germinação e no vigor de sementes de arroz. Pesquisa Agropecuária Tropical, v.18, n.1, p.127-139, 1988. http://www.revistas. ufg.br/index.php/pat/article/view/2558/2530

WILDE, G.; ROOZEBOOM, K.; AHMAD, A.; CLAASSEN, M.; GORDON, B.; HEER, W.; MADDUX, L.; MARTIN, V.; EVANS, P.; KOFOID, K.; LONG, 
J.; SCHLEGEL, A.; WITT, M. Seed Treatment Effects on Early-Season Pests of Corn and on Corn Growth and Yield in the Absence of Insect Pests. Journal of
Agricultural and Urban Entomology, v.24, n.4, p.177193, 2007. http://www.bioone.org/doi/abs/10.3954/15235475-24.4.177? journalCode=jaue 